\title{
Iron Tablets or Gallstones? Iron Overdose in Situs Inversus
}

\author{
Jasmin Gosen $\mathrm{BA}^{1}$, Chelsea Hayman $\mathrm{BS}^{2}$, Steven Offerman ${ }^{3}$ and Leonard Ranasinghe ${ }^{4 *}$ \\ ${ }^{1}$ Department of Medicine, California Northstate University, USA \\ ${ }^{2}$ College of Medicine, California Northstate University, USA \\ ${ }^{3}$ Emergency Department, Kaiser Permanente Hospital, USA \\ ${ }^{4}$ Professor of Emergency Medicine, California Northstate University, USA
}

*Corresponding author: Leonard Ranasinghe, Professor of Emergency Medicine, California Northstate University, Elk Grove, California, USA.

To Cite This Article: Leonard Ranasinghe, Iron Tablets or Gallstones? Iron Overdose in Situs Inversus. 2020 - 8(2). AJBSR.MS.ID.001260. DOI: 10.34297/AJBSR.2020.08.001260

Received: 眥 March 10, 2020; Published: March 19, 2020

\section{Case Presentation}

A 38 year-old male with a history of psychiatric illness and methamphetamine abuse presented to the emergency department (ED) reporting intentionally taking cyclobenzaprine, alprazolam, and iron tablets. He could not provide history regarding exact dosages or time of ingestion. An abdominal x-ray showed multiple radio-opacities in the right upper quadrant. (Panel $\mathrm{A}$ ) The location and exact nature of these opacities was initially unclear. A chest $\mathrm{x}$-ray suggested situs inversus. (Panel B) The foreign bodies were then found to be iron tablets located in the stomach. Whole bowel irrigation (WBI) with polyethylene glycol was instituted to decontaminate the gastrointestinal tract. Following WBI, the gastrointestinal tract was clear of all radiopaque tablets. (Panel C) The patient had no complications following the procedure and remained stable after the remaining iron tablets were removed.

\section{Discussion}

Opaque foreign bodies in the upper right quadrant on x-ray likely to be iron tablets in the stomach suggesting situs inversus. Situs inversus is an rare inherent condition consisting of inversion of the major organs from their normal positions in the body. Abdominal and thoracic organs are transposed and seen as a mirror image of normal anatomy. It occurs in approximately 1 in 10,000 persons in the population $(0.01 \%)$. Some patients have comorbid conditions such as primary ciliary dyskinesia, congenital heart disease, renal disorders and biliary atresia that cause far more complications. Patients with isolated situs inversus are usually asymptomatic and have a normal lifespan [1]. Prior to diagnosis of this anatomic abnormality in patients, situs inversus may cause confusion for physicians thus affecting diagnostic studies and procedures. Situs inversus complications most often occur in undiagnosed patients with abdominal pain, where left lower quadrant pain can be confused with diverticulitis instead of appendicitis. It is important to recognize situs inversus in order to prevent surgical confusion or complications by failing to identify the reversed anatomy in procedures such as appendectomy or cholecystectomy. There have been no documented cases of a patient with situs inversus with an iron overdose [2] .

Intentional overdose of iron tablets in adults is uncommon but more commonly seen in accidental ingestion in children under 5 years old. It adults, iron overdose is more commonly seen with multiple drug ingestion and can lead to GI, cardiovascular, metabolic, hepatic and central nervous system toxicity. Acute iron poisoning is generally characterized in five overlapping phases. The first phase is the gastrointestinal phase starting at 30 minutes to 6 hours after ingestion, and is characterized by abdominal pain, vomiting, diarrhea, melena, shock - causing death in this phase and metabolic acidosis. The iron tablets cause direct injury to the GI mucosa, leading to these symptoms. Patients with mild to moderate toxicity generally do not go beyond this phase [3].

From 6 hours to 24 hours is the latent phase, where symptoms are relatively stable and the patient appears to recover. However, close monitoring is essential in case of severe iron poisoning and clinicians should watch out for signs of poor perfusion, hyperventilation, and oliguria.d This phase is often transient or may not occur with severe poisoning. Shock and metabolic acidosis can 
occur from 6 to 72 hours after ingestion, which may lead to multisystem organ failure. Hepatotoxicity and hepatic necrosis generally develop a few days after ingestion if a patient is not adequately treated. Liver failure is the second most common cause of death. Two to eight weeks after ingestion, patients can develop bowel obstruction from GI scarring [4]. When a significant number of iron pills are seen on abdominal radiograph, a whole bowel irrigation (WBI) should be performed to prevent uptake of the additional iron in the gut. Treatment is ended when the patient is clinically stable, has a clear rectal effluent, and there is an absence or a decreased number of iron tablets seen on repeat imaging [5] (Figure 1\& 2 \& 3).

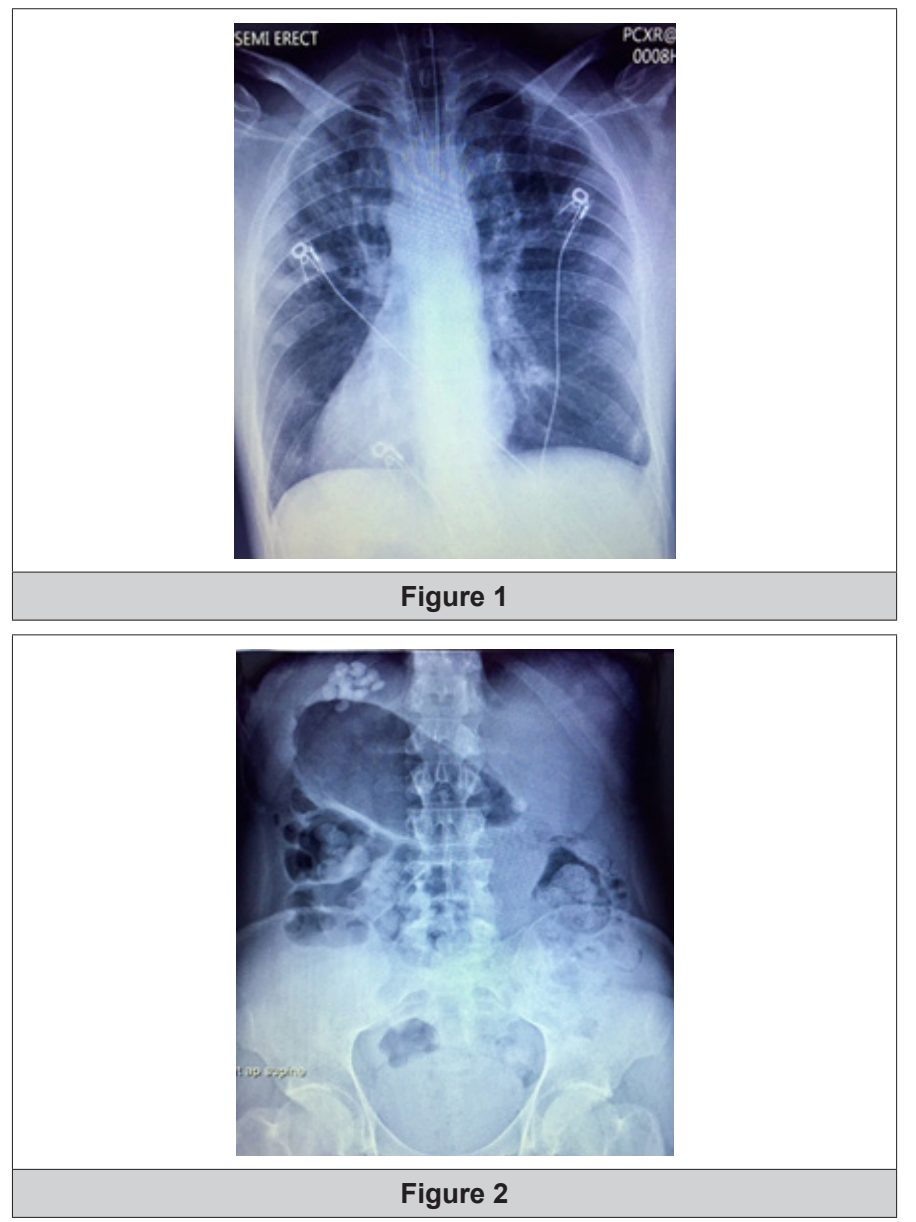

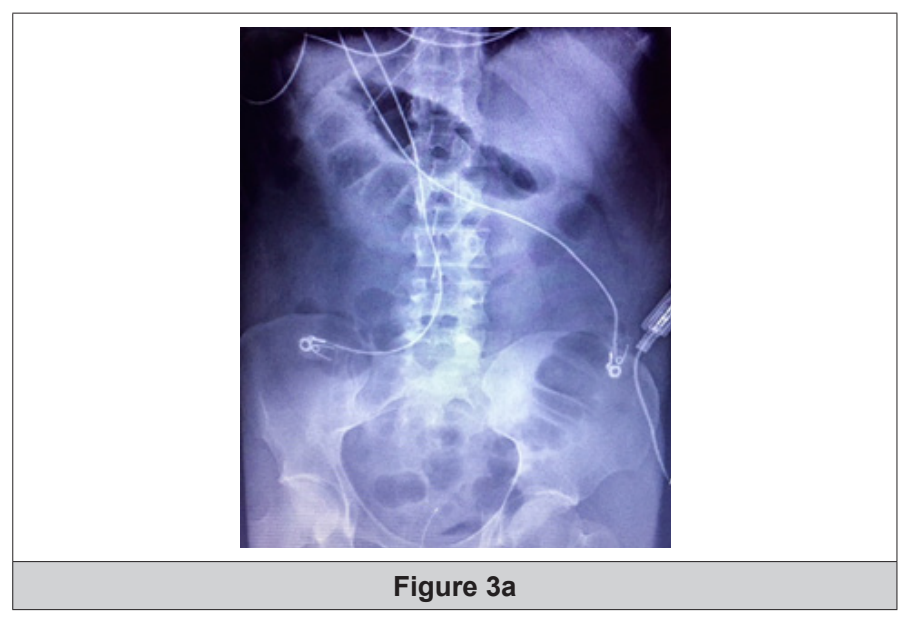

WBI may be indicated in cases of potentially dangerous poisoning where the toxin is not amenable to activated charcoal. To perform WBI irrigation, a nasogastric tube should be placed and PEG solution titrated to a goal rate of 1-2 litres per hour. Due to patient intolerance, 2 litres per hour is rarely attainable. WBI should not be performed in cases where the airway is at risk or unprotected.

\section{References}

1. Cotran RS, Kumar V, Robbins SL (1989) (4 ${ }^{\text {th }}$ Ed.) WB Saunders Co Robins pathological basis of disease, Philadelphia, USA, p. 777.

2. Abhilash KP, Arul JJ, Bala D (2013) Fatal overdose of iron tablets in adults. Indian J Crit Care Med 17(5): 311-313.

3. Reynolds, Louis G (1989) "9 Diagnosis and Management of Acute Iron Poisoning." Baillière’s Clinical Haematology 2(2): 423-433.

4. Tenenbein M (1198) "Toxicokinetics and Toxicodynamics of Iron Poisoning." Toxicol Lett 95(35): 653-656.

5. Tenenbein Milton (1987) "Whole Bowel Irrigation in Iron Poisoning." The Journal of Pediatrics 111(1): 142-145. 